\title{
Aproximación a la evolución de los cambios en los servicios de salud en Cuba
}

\section{Approach to the evolution of changes in health services in Cuba}

\section{DraC. Luisa I ñiguez Rojas}

Centro de Estudios de Salud y Bienestar Humano, Universidad de La Habana. La Habana, Cuba.

\begin{abstract}
RESUMEN
Partiendo de una caracterización general de los procesos impulsados desde mediados de la década del 90 del pasado siglo, encaminados a la recuperación del sector de la salud, y centrados en mejorar la calidad de los servicios, la eficiencia, la efectividad, y mantener la equidad, se describe la evolución de indicadores fundamentales de servicios, de recursos humanos y de infraestructuras en salud. Tomando como substrato los anteriores resultados, se exponen los objetivos y contenidos esenciales de las actuales transformaciones del Sistema Nacional de Salud basado en procesos de reorganización, concentración y regionalización de los servicios, para discutir implicaciones de las reestructuraciones en la distribución territorial, con énfasis en las tensiones entre racionalidad y equidad de acceso real o percibido.
\end{abstract}

Palabras clave: Servicios de salud, transformaciones, Cuba.

\section{ABSTRACT}

The general characterization of the processes that have been prompted since the mid 90's, aimed at the health sector recovery and at the improvement of the quality of service, the efficiency and the effectiveness of the system, and at keeping equity, was taken as basis for the description of the evolution of fundamental indicators of service, human resource and health infrastructure in this article. The 
essential objectives and contents of the present changes in the national health care system aided by reorganization, concentration and regionalization of services were explained in order to discuss the implications of restructuring in the territorial distribution, emphasizing controversies between rationality and real or perceived access equity.

Key words: Health services, changes, Cuba.

En la actualidad Cuba se enfrenta a diferentes procesos, que se centran en el análisis de los problemas más acuciantes del país, y han sido aprobados los lineamientos de la política económica y social. ${ }^{1}$ Varios cambios han ocurrido y se avizoran en esta perspectiva, y dentro de ellos, nuevas transformaciones en el Sistema Nacional de Salud (SNS).

A mediados de la década del 90, en el Ministerio de Salud Pública, se inicia un nuevo momento de transformación del sector, con el objetivo de definir las principales líneas estratégicas y programas, que permitirían desarrollar un proceso de consolidación y modernización del sistema, con nuevos métodos y estilos de trabajo. Desde entonces se planteaba que el gasto en salud se incrementaba, y en consecuencia se limitaban otras actividades y ramas presupuestadas. ${ }^{2}$

A inicios de la pasada década, fueron implementados numerosos programas sociales, inscritos en lo que se denominó "Batalla de Ideas", dentro de los cuales, se diseñaron acciones para la recuperación y perfeccionamiento del sistema, integradas en la llamada "Revolución en el sector salud". A finales del 2010, se anunció el despliegue de un proceso de "Reorganización, concentración y regionalización de los servicios de salud", que tenía entre sus antecedentes, el discurso pronunciado en diciembre del 2009 por el Presidente del Consejo de Estado de Cuba, y en el que afirmaba que "...sin afectar la calidad de la salud, que se brinda gratuitamente a todos los ciudadanos, e incluso mejorándola, es posible reducir de modo apreciable los gastos." ${ }^{3}$

Teniendo en cuenta estas premisas, se intentará una aproximación contextualizada de la evolución reciente y la situación actual de los servicios de salud en el país, mediante la formulación de algunas interrogantes: ¿Cómo se integra la revolución del sector iniciada en la década pasada, a las transformaciones que se realizan en la actualidad?, ¿Cuáles pueden ser los principales obstáculos en la consecución de los nuevos objetivos?

\section{DETERIORO DE LOS SERVICIOS Y REVOLUCIÓN EN EL SECTOR DE LA SALUD}

Los efectos primarios de la profunda crisis económica que enfrentó el país a inicios de la década del 90, y sus efectos secundarios, provocaron el deterioro infraestructural de instalaciones, serias carencias de recursos materiales, deficiencias en el desempeño profesional, falta de capacitación gerencial para la conducción del sistema de salud a nivel de las unidades, acompañadas de un 
incremento de las demandas de atención en hospitales, y la insatisfacción creciente de la población, incluyendo la atención a urgencias. ${ }^{1,4}$ Los resultados de la encuesta nacional sobre satisfacción de los usuarios con los servicios de atención médica, del Ministerio de Salud Pública, 1990, mostraban una situación nada favorable: aproximadamente el $70 \%$ de la población se encontraba medianamente satisfecha y el 10,6 \% insatisfecha, situación que se agravó en los años subsiguientes.

\section{Al respecto Suárez, 1997 plantea:}

...El impacto de la crisis en los servicios de salud presenta retos de difícil solución en un país con un universal y gratuito sistema de salud, una población acostumbrada a utilizar ampliamente los servicios, incluyendo los de tecnología avanzada y una realidad en que el acceso a créditos externos por una parte, y a mercados de medicamentos, insumos y equipos médicos por la otra, se dificulta por efectos del bloqueo, lo que encarece y limita su adquisición... ${ }^{5}$

Desde mediados de la década del 90, el Ministerio de Salud Pública iniciaba estudios con el fin de diagnosticar la situación del SNS, y trazar estrategias para la recuperación del sector, centradas en mejorar la calidad de los servicios, la eficiencia, la efectividad, y mantener la equidad. ${ }^{1}$

Se definían como líneas prioritarias: la reorientación del sistema de salud hacia la atención primaria, la revitalización de la atención hospitalaria, la reanimación del trabajo de los programas de tecnología de punta e institutos de investigaciones, el desarrollo del Programa de Medicamentos y de Medicina Natural y Tradicional, así como la atención priorizada a objetivos tales como servicios de urgencia, estomatológicos y de ópticas. Se consideraban de máxima prioridad los programas de atención materno infantil, de enfermedades crónicas no transmisibles, de enfermedades transmisibles y del adulto mayor. Los llamados "Programas de la Revolución en Salud", fueron estructurados a inicios de la pasada década, con los objetivos de perfeccionar el sistema en general y la atención primaria en particular, y se retomaron en los procesos de reformas ya iniciados. En ellos se reafirmaba la necesidad de cambios en la estructura organizativa del sistema, en especial en la atención ambulatoria, la organización de los servicios y los programas de salud. ${ }^{6}$

Una de las acciones en la concreción de tales empeños, fue la organización del "Programa de Reconstrucción y Modernización de los Policlínicos", que se proponía el acercamiento de los servicios a la población, la adecuación de estos servicios a la situación de salud de cada lugar, la formación y el perfeccionamiento continuo de los recursos humanos, así como al mejoramiento e introducción de nuevas tecnologías. ${ }^{5}$

Se reparaban los policlínicos, y se ampliaban sus servicios mediante la transferencia tecnológica de los niveles secundarios y terciarios (hospitales generales y especializados), hacia el primer nivel de atención, en una magnitud sin precedentes hasta entonces. ${ }^{7}$

Los policlínicos eran dotados como promedio de 20 servicios, que sobrepasaban los 30 en algunos de ellos, tales como rehabilitación, radiología, ecografías, optometría, endoscopia, trombolísis, servicios de urgencia, traumatología, laboratorio clínico, planificación familiar, urgencias odontológicas, atención materno infantil, inmunización, atención a los diabéticos y a las personas de edad avanzada, además de consultas en las especialidades de medicina interna, pediatría, obstetricia y ginecología, dermatología, psiquiatría y cardiología. La reparación y renovación o introducción de equipamientos tecnológicos, beneficiaba también a los 
servicios secundarios incluidos en el otro "Programa de Reconstrucción y Modernización de Hospitales."

En paralelo, se producía el cierre paulatino de consultorios del médico y la enfermera de la familia, se agrupaba la población atendida en varios consultorios en uno, y si bien se mantenía la cobertura de atención en el $100 \%$, se multiplicaba la población a atender, con la consecuente recarga del trabajo de los médicos y enfermeras que afectaba seriamente la atención antes prestada y, en especial la percepción sobre esta. No variaba la prioridad concedida a algunos programas, como el de atención materno infantil (PAMI), y se debilitaban las acciones promocionales y el trabajo de terreno, funciones intrínsecas a este programa (visitas programadas del médico y la enfermera a las viviendas donde residía población vulnerable dentro de los programas de atención).

La reducción de los consultorios, y la compactación de los servicios brindados por el médico y la enfermera de la familia, se ha asociado a la salida masiva de médicos para cumplir misiones de colaboración en varios países, mientras, otras opiniones destacan como determinantes los graves problemas organizativos que confrontó el Programa del Médico y la Enfermera de la Familia (PMEF), y no la falta de médicos en este nivel de atención. ${ }^{8,9}$ Se integró a esta reducción, el mantenido deterioro infraestructural de consultorios, y la carencia de materiales de trabajo.

En consecuencia, fueron crecientes las insatisfacciones de la población, asociadas a la inestabilidad de los servicios, al tiempo de espera de atención, a la ausencia del médico por estar cumpliendo otras funciones administrativas o tareas docentes nuevas, en el marco de la "adaptación" a un nuevo médico en el territorio. Esta situación se complica por el cierre total o parcial de instituciones hospitalarias y por ende de servicios de mayor complejidad, en el marco del reequipamiento de hospitales o de policlínicos en reparación. La percepción era aún más negativa por el referencial de atención anterior, máxime cuando el consultorio debía ser la puerta de entrada al sistema de atención de mayor complejidad y especializada, muchos de los cuales estarían ahora disponibles en los policlínicos.

La reducción observada en el número de consultas médicas totales a inicios de la década pasada, llegó a ser de dos consultas menos por habitante entre el 2002 y el 2006, descenso más notable incluso que el producido en la década del 90 . Vale la pena comentar que el descenso fue más agudo que el experimentado en 1993, año de incremento de la incidencia de algunas entidades nosológicas, que exigieron de la intensificación de pesquisajes activos que pueden haber influenciado en la disminución de consultas (Fig. 1).

Teniendo en cuenta esta situación, como parte de la reorganización de la atención primaria, a partir de 2008 , cuando la población tributaria a un consultorio llegaba a ser hasta de 2500 personas, se estableció, con carácter transitorio, una tipología (I, II y III) basada en horarios de funcionamiento, y dotación de recursos humanos según factores de localización y acceso a otras instituciones de salud. El tipo I funcionaba ocho $\mathrm{h}$ diarias, media jornada los sábados y un día a la semana hasta horas de la noche, con un equipo multidisciplinario que atendía un promedio de entre dos mil 500 y tres mil personas. El tipo II con personal de enfermería ocho $h$, garantizando las labores orientadas por el médico a la población y el tipo III, con médico y enfermera las $24 \mathrm{~h}$ del día, localizados en los lugares más apartados. 


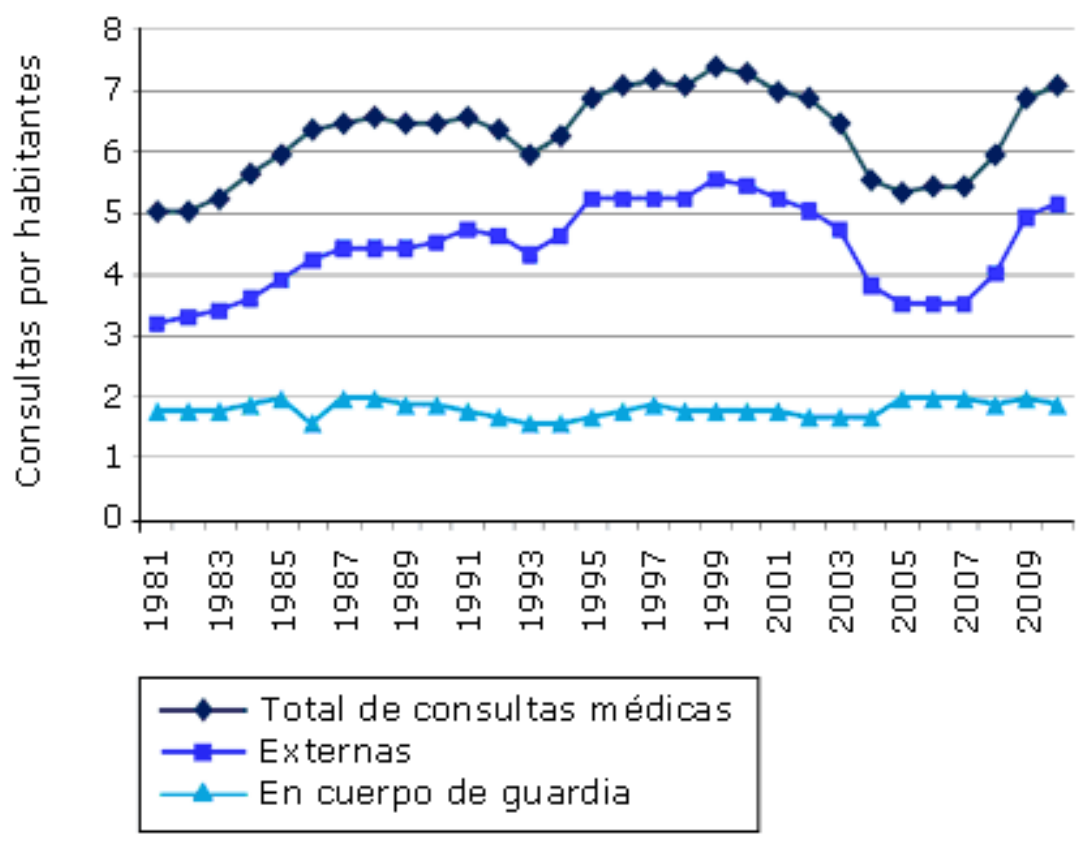

Fuente: Anuario Estadístico de Salud 2009 y 2010. Dirección Nacional de Registros Médicos y Estadísticas de Salud (DNRME), MINSAP.

Fig. 1. Consultas externas y de cuerpos de guardia.

La recuperación del número de consultas externas a partir del 2008, sugiere la influencia positiva de tal reorganización.

Coincidente con estas inflexiones, las actividades quirúrgicas seriamente afectadas desde la década del 90, mantuvieron una tendencia a su disminución para llegar en el 2006 a menos 58000 cirugías en relación con el año anterior, hecho que sugiere la pérdida de capacidades del sistema para satisfacer la demanda de este servicio, en el marco de los programas de reparación y modernización en marcha en las instituciones, sin desconsiderar las limitaciones de recursos en estas prácticas médicas.

Algunos indicadores de instalaciones destinadas al cuidado de grupos vulnerables, como embarazadas y ancianos, variaron la disponibilidad de sus servicios, donde puede también haberse producido una eventual reducción de la demanda. Los albergados en hogares de ancianos en régimen interno, se mantuvieron estables durante la década del 90 y los primeros años de la década del 2000, para experimentar una ligera reducción mantenida a partir del 2003 del orden de poco menos de 300 ancianos beneficiados; por el contrario, los de régimen seminterno, experimentaron un intenso crecimiento que a inicios de la década del 2000 duplicaba la cifra de ancianos en hogares con más de 3000 beneficiarios (Fig. 2). 


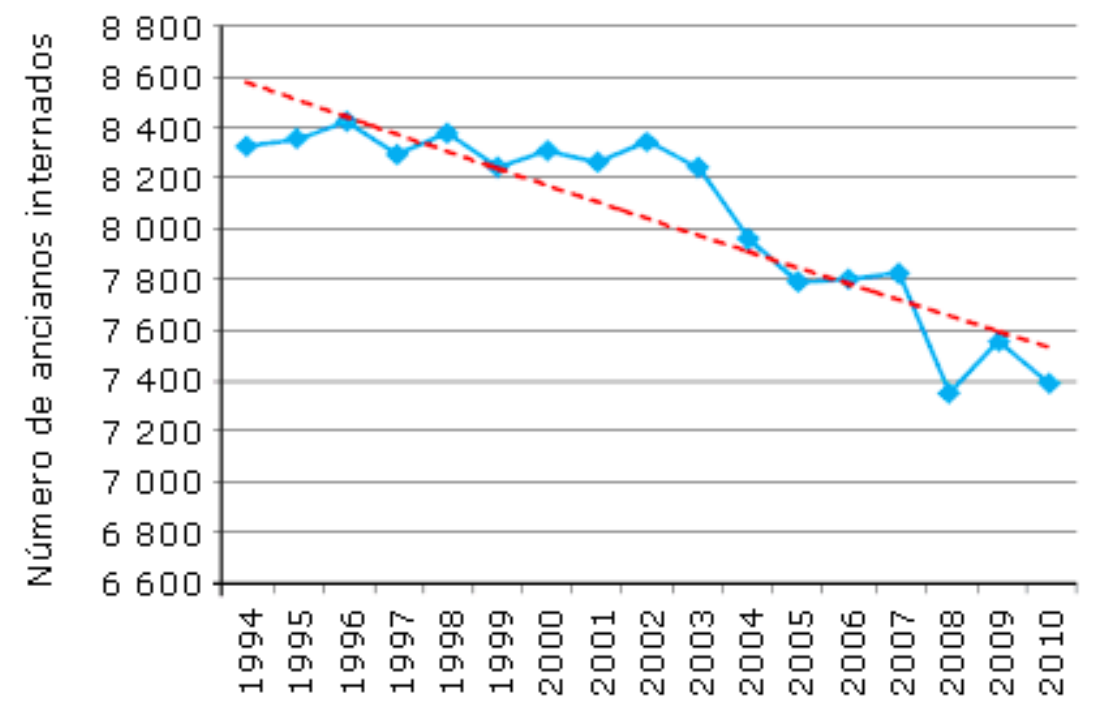

Fuente: Anuario Estadístico de Salud 2009 y 2010. DNRMES. MINSAP.

Fig. 2. Ancianos en hogares del MINSAP.

Otros recursos y servicios para estos grupos de población continuaban brindándose en instituciones tales como las casas de abuelos, de atención integral al adulto mayor y variantes locales de atención, a lo cual se integraba la asistencia social en la propia residencia de los adultos en condiciones de más elevada vulnerabilidad social.

Los hogares maternos se organizan por la necesidad de acercar a las gestantes a los centros hospitalarios, en ellos además de la atención prenatal, se cuida la nutrición y se brinda educación sanitaria con prioridad a la promoción de la lactancia materna y el cuidado del niño. En estas instituciones se incrementó el ingreso de embarazadas, con un descenso a partir del 2002, que como en anteriores descripciones, alcanzó sus menores cifras en el 2006, con poco menos de 7000 mujeres internadas, para experimentar a partir de entonces, un incremento exponencial que alcanzó en el 2009 su máximo valor, para un indicador de 50,5 ingresadas en hogares maternos por 100 nacidos vivos (incluye las embarazadas en hogares con régimen seminterno). En el año 2010 este valor es sobrepasado hasta el 52,8 de ingresos por 100 nacidos vivos (Fig. 3).

Ello puede asociarse a diferentes factores entre los que estarían la adopción de una alternativa en forma de régimen de seminternado, el ascenso del número de nacimientos en los dos últimos años del periodo que se analiza, y a la prioridad que el PAMI mantiene a este procedimiento de probada efectividad.

La prioridad concedida al sector de la salud desde 1959 determinó un incremento notable en la ejecución del presupuesto y un particular ascenso en la primera década del presente siglo, más intenso a partir de 2006, coherente con la consolidación de los programas de la revolución antes citados, para llegar en el $2009,{ }^{10}$ a su cifra más elevada de 440,30 pesos por habitante (Fig. 4). 


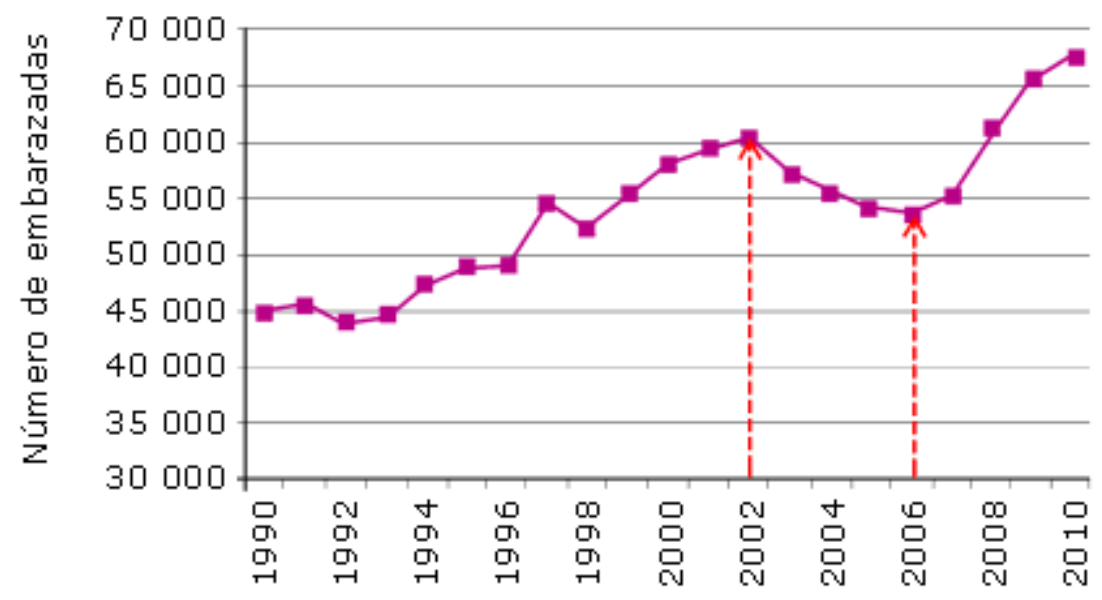

Fuente: Anuario Estadistico de Salud 2009 y 2010. DNRMES. MINSAP.

Fig. 3. Embarazadas en hogares maternos.

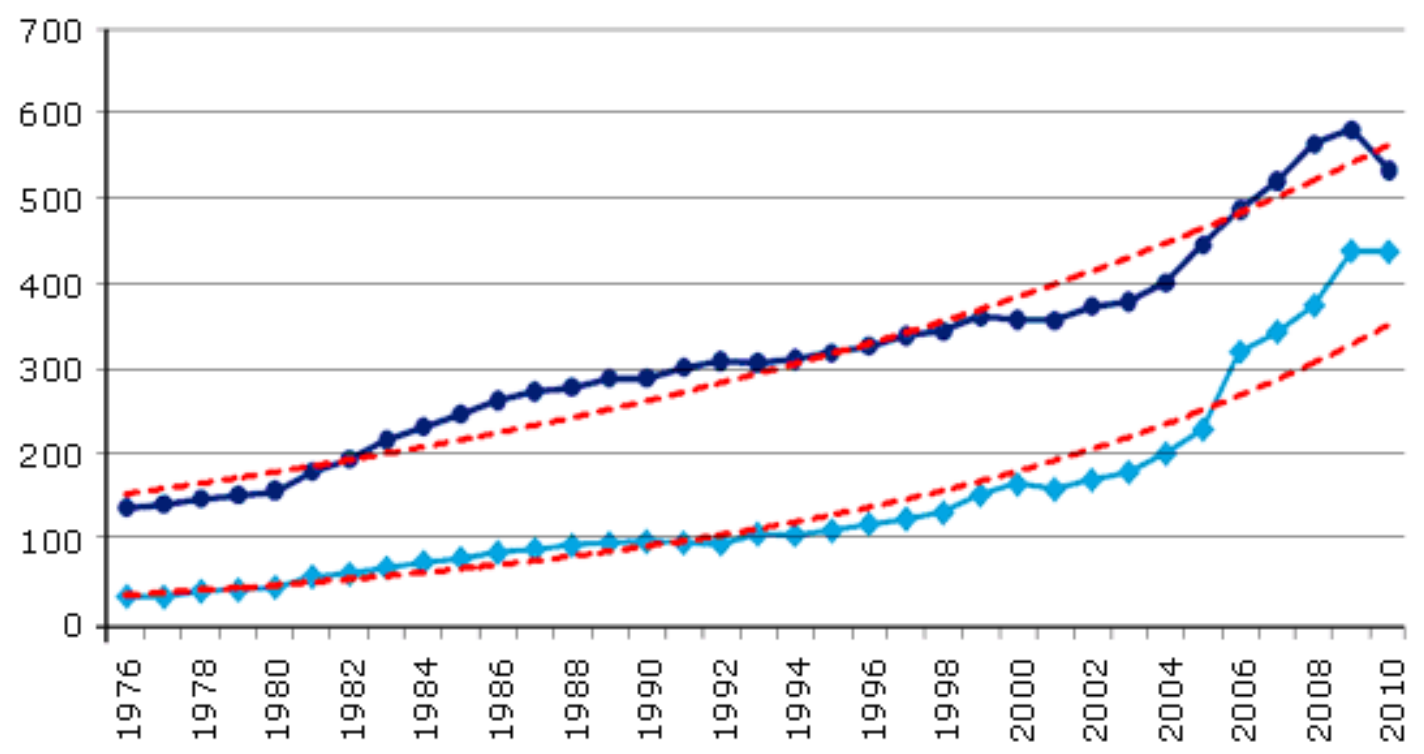

$$
\begin{aligned}
& \longrightarrow-\text { Gastos en peso por habitante } \\
& --- \text { Trabajadores de la salud (miles) } \\
& \longrightarrow \backsim \text { Exponencial (gastos en peso por habitante) }
\end{aligned}
$$

Fuente: Anuario Estadístico de Salud 2009 y 2010. DNRMES. MINSAP.

Fig. 4. Evolución del gasto en salud en pesos por habitante y de los trabajadores de la salud 1976-2010.

El incremento observado en el número de trabajadores de la salud en la década del 80 por efecto de la implementación del PMEF, es a partir del 2002 y en especial en el 2006, mucho más intenso, hecho explicado en lo esencial por la incorporación de trabajadores vinculados a los nuevos o ampliados servicios en los policlínicos, y por 
haberse concebido un proceso docente continuo de este profesional, de forma que al terminar el primer año de estudio, se convertían en trabajadores, sin descontar el discreto aumento de salario aplicado a mediados del 2005.

Así, la formación de recursos humanos en salud con un notable crecimiento en la década del 80, experimentó una reducción desde finales de la década del 90, para asumir un notable incremento a partir del 2006 y alcanzó en el 2010 la cifra de 26 600 graduados de la educación superior de las diferentes especialidades. Este hecho es explicado en lo fundamental por el número de profesionales formados en tecnología de la salud, ${ }^{10}$ que incluso según el esquema docente aplicado, se incorporaban como trabajadores y percibían salarios, al finalizar el primer año de estudio (Figs. 5 y 6 ).

La estructura de los trabajadores de la salud según ocupación en el 2009, muestra que los indicadores más elevados se obtenían en las ocupaciones antes mencionadas con 119 técnicos medios por 10000 habitantes y 86,5 en personal de enfermería por 10000 habitantes. En ese propio año se contaba con 66,6 médicos por 10000 habitantes, y 10,3 estomatólogos por 10000 habitantes. Al cierre del año 2009, se registraban 74880 médicos en activo, de los cuales el $73 \%$ estaban especializados. El número mayor de médicos se correspondía a los formados en medicina general integral ( $45 \%$ del total), con el $75 \%$ de ellos especialistas, seguido de los dedicados a la organización y administración en salud (aproximadamente el $20 \%$ del total) con solo $5 \%$ de especialistas. Otras de los médicos más representados se dedicaban a la medicina interna, la ginecoobstetricia, y la pediatría. ${ }^{10}$

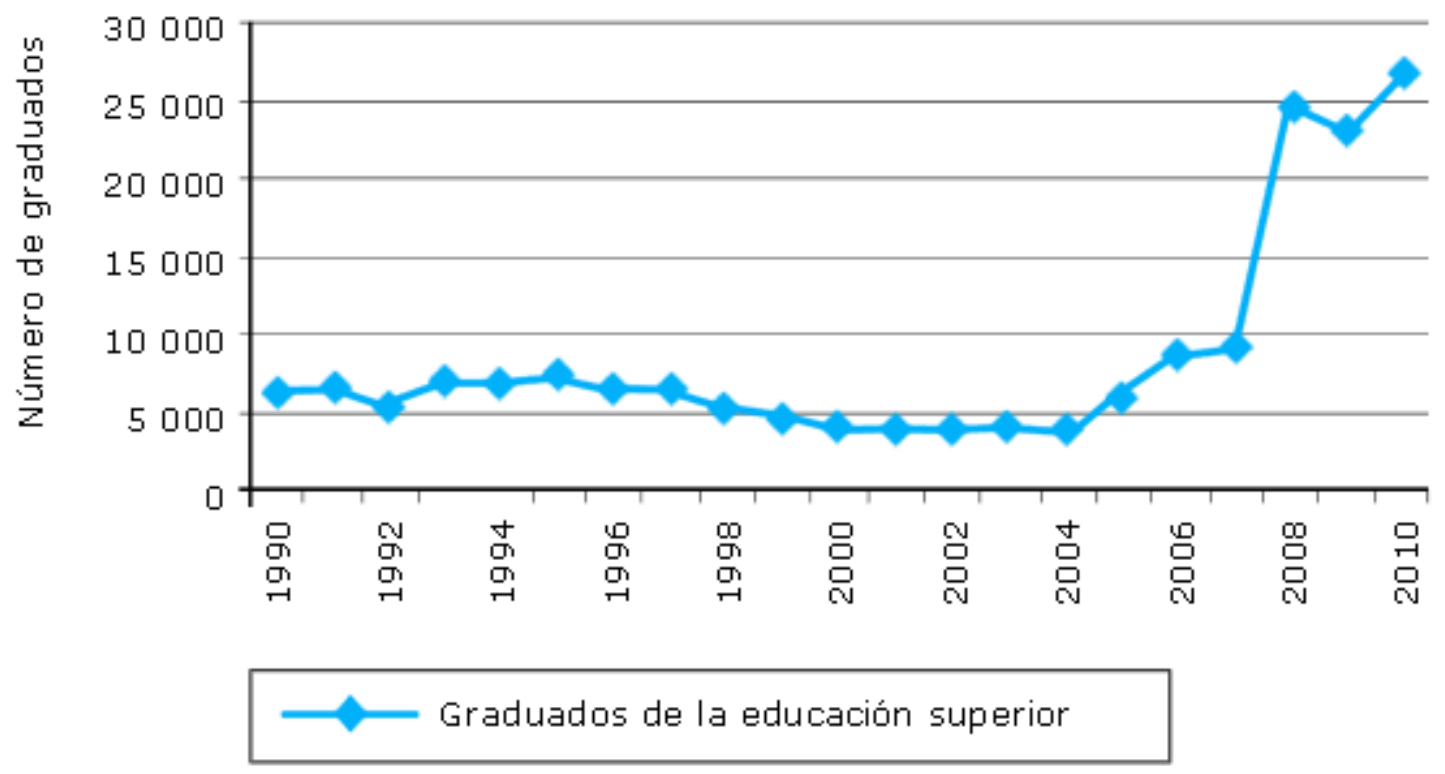

Fuente: Anuario Estadístico de Salud 2009 y 2010. DNRMES. MINSAP.

Fig. 5. Formación de recursos hum anos en salud, 1990-2010. 


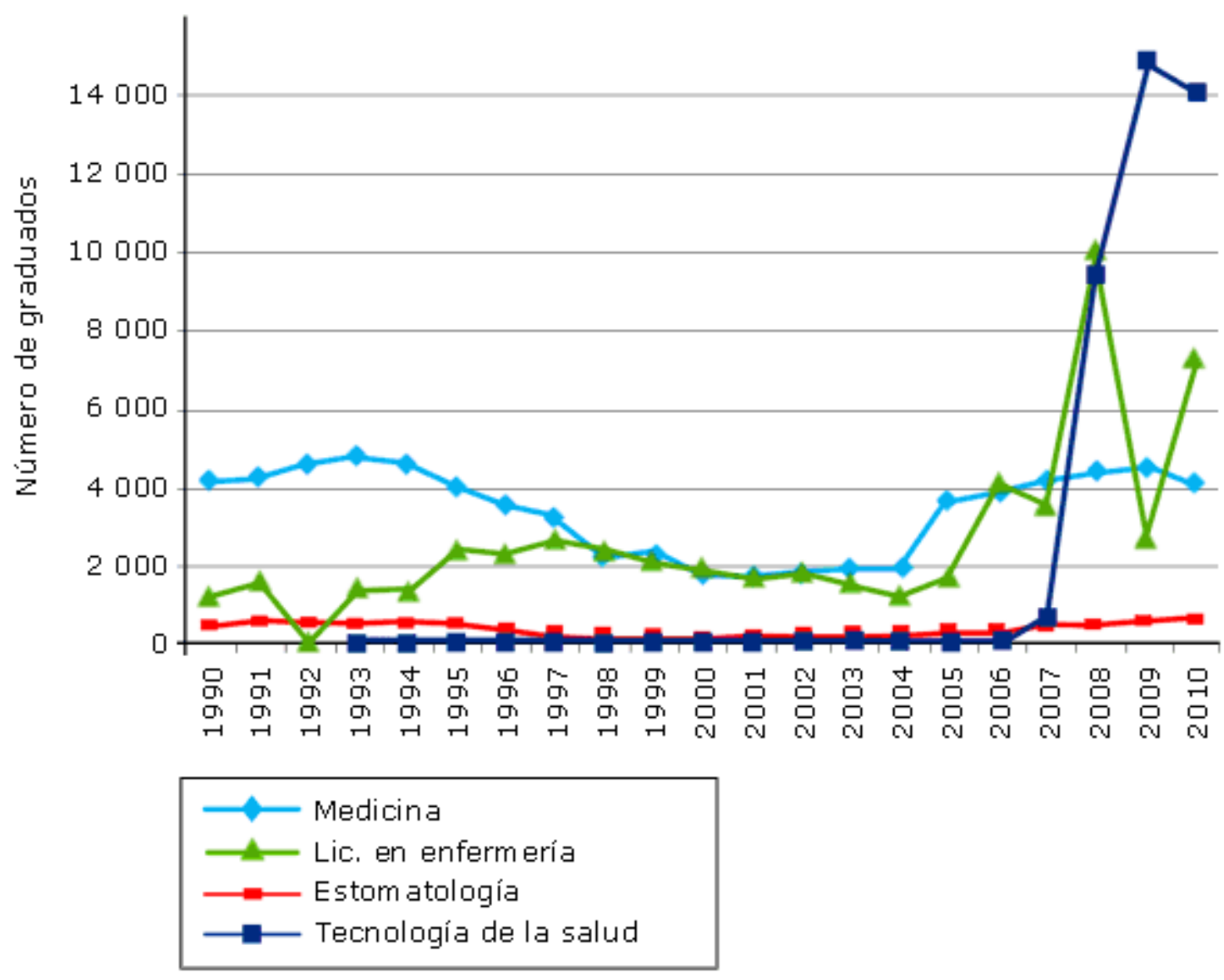

Fuente: Anuario Estadístion de Salud 2009 y 2010. DNRMES. MINSAP

Fig. 6. Formación de recursos hum anos en salud por especialidades.

Son regularidades notables de las anteriores descripciones el movimiento de indicadores de servicios a inicios de la primera década del 2000 , coincidente con el comienzo de la ejecución de los Programas de la Revolución en el sector y el incremento del crecimiento del número total de trabajadores, del gasto por habitante, y de la formación de trabajadores en algunas especialidades, en el marco del mantenido incremento del indicador de médicos por habitantes que para el 2010 alcanzó la cifra de 68,1 médico por 10000 habitantes. ${ }^{11}$

Las inflexiones observadas, disminución a inicios de la década del 2000, y recuperación a partir del 2006, sugiere la influencia de los programas en marcha, con expresiones diferenciadas en los territorios, que se mueven entre la reducción de la oferta de servicios, o por el contrario de la demanda, según la etapa de reconstrucción y modernización de las instituciones de salud.

Como ha sido notado en diversas publicaciones, indicadores de salud de relevancia internacional, tales como mortalidad infantil, mortalidad en menores de 5 años, incidencia de enfermedades infecciosas y otros, en general no se deterioraron, e incluso mejoraron, lo cual se explica no solo por la prioridad que los respectivos programas de atención han mantenido desde la propia creación del SNS, como por avances, aunque de forma diferenciada según grupos en espacios sociales, en dimensiones de condiciones de vida en la última década. 
Por otra parte, el impacto positivo de los programas de reconstrucción y modernización de los policlínicos y el incremento de los servicios en estos, puede apreciarse en el desplazamiento proporcional de las consultas hacia ellos. En el 2010 , en el policlínico se realizaron cerca del $90 \%$ de las consultas externas totales y el $65 \%$ del total de las consultas de urgencia. ${ }^{11}$

Un último y delicado aspecto, participa en la trama de componentes y procesos en los cuales evolucionaron los programas para la recuperación de la calidad de los servicios de salud en el país. Se trata de componentes más difíciles de medir, conformados en la dualidad subjetivo-objetiva de estos procesos que provocan efectos secundarios tales como la desmotivación hacia el trabajo y la percepción de desigualdad de los trabajadores del sector. Aunque es totalmente cierta la necesidad de profundizar en las motivaciones espirituales de los trabajadores para conseguir el pleno cumplimiento del encargo social de la salud pública, ${ }^{5}$ también es necesario profundizar en el análisis de las condiciones materiales de vida y laborales de los profesionales y en general de los trabajadores del sector.

Es bien conocido que la mayoría de los médicos cubanos que no han cumplido misiones internacionalistas, o se benefician por algunas otras fuentes de ingresos suplementarios, incluso no provenientes del trabajo, como ayudas familiares y remesas, son en general ciudadanos que experimentan similares limitaciones económicas, a los de otros trabajadores del país (profesionales o no), lo cual se concreta en la reconocida brecha entre oferta y demanda, o demanda sin oferta, y tantas otras limitaciones en la satisfacción de necesidades básicas, tradicionales o de nuevos satisfactores de viejas necesidades. Estas situaciones aunque no son exclusivas de Cuba, si son infrecuentes en otros países, donde profesiones de elevada representación social como médicas, enfermería, de especialistas en rehabilitación, entre otros, con frecuencia se distinguen como un estrato en ventajas de ingresos.

\section{TRANSFORMACIONES ACTUALES EN EL SISTEMA NACIONAL DE SALUD. DISTRI BUCI ÓN Y EQUI DAD EN FOCO}

El Ministerio de Salud Pública elaboró en fecha reciente un documento sobre las transformaciones del SNS, del que nos valdremos para analizar las actuales propuestas. ${ }^{12}$

El documento considera que las trasformaciones permitirán:

...el uso más eficiente y racional de los cuantiosos y costosos recursos de que disponemos, especialmente los tecnológicos, así como su permanente disponibilidad, lo que garantizará la sostenibilidad de los servicios de salud.

Aunque el móvil inicial es sin duda económico, es evidente que en estas transformaciones, se consideran otros elementos, y tal como plantea el documento citado, las transformaciones pretenden asumir la "necesidad impostergable de alcanzar niveles superiores de eficiencia y calidad". ${ }^{12}$ De forma coherente, el primero de los lineamientos del sector de la salud contenido en los lineamientos de la política económica y social del país aprobado en fecha reciente plantea "potenciar la calidad de los servicios y la utilización eficiente de los recursos, con la eliminación subsecuente de los gastos". Según cifras oficiales durante la década del 2000, la 
ejecución del presupuesto no descendió y para el 2010 se observa una reducción de poco más de 10 millones de pesos.

De forma explícita se establece que la reorganización de las estructuras de dirección deberá responder a las necesidades de la situación de salud en cada territorio, con el personal estrictamente necesario y con la calificación y experiencia que permita lograr eficiencia en el trabajo. Junto a la reducción del número de trabajadores que se prevé, se considera que los que dirijan, deberán haber transitado por el sistema, antes de ocupar un cargo de dirección en cualquiera de los niveles de atención, y poseer la calificación y experiencias necesarias. También asociado a la calificación, se resalta la necesidad de que los médicos apliquen el método clínico y el epidemiológico, como principal garantía de la calidad de los servicios de salud, coherente con la pérdida de atención a estos métodos, y la prioridad dada por los médicos y la población a las tecnologías de diagnóstico.

La regionalización de los servicios se considera la vía para mantener los logros alcanzados en el orden técnico y material del sector de la salud, ante la situación económica que enfrenta el país, y se sustenta en una redistribución territorial de servicios, que se ejemplifica en que:

...un equipo preste su servicio en un área de salud, en un municipio o en la cabecera provincial, en correspondencia con el nivel de actividad existente y allí acuda la población a recibirlo, lo que permite, además, que los especialistas y técnicos de mayor preparación brinden el servicio, garantizándose mayor calidad. ${ }^{12}$

Se asiste así, al análisis y propuestas de redistribución de los servicios, en especial en la atención primaria, la mayoría de los cuales fueron implementados, en años recientes en el marco de la revolución en salud, tales como los de rehabilitación, endoscopia, regulación menstrual, imaginología, cirugía menor y de urgencia, entre otros, y se orienta que en los casos en que se demuestre la subutilización, sean regionalizados, para ampliar la cantidad de beneficiarios y el número de asentamientos, o municipios que integran el área que tributa a los servicios en cuestión.

Los procesos de regionalización, representan una de las principales readaptaciones al criterio esgrimido en la revolución del sector desplegada en la pasada década, acercar la población a los servicios-, ante la necesidad de una distribución cuya racionalidad asegure el mantenimiento de algunos de ellos. Si bien es cierto como se plantea, que esta es una forma de asegurar la mayor calidad, el aspecto de accesibilidad se constituye en un factor que puede restringir los logros esperados de esta regionalización, en términos de satisfacción y otros.

En relación con la distribución de los policlínicos, se propone el reanálisis del nivel de actividad y de los recursos humanos necesarios en aquellos que atienden menos de 5 mil habitantes, y en el caso de comprobarse la subutilización de recursos o servicios, estos serán convertidos en consultorios médicos, redefiniéndose sus funciones según el universo poblacional a atender. Es este otro delicado criterio que puede colocar en riesgo la equidad distributiva.

En los hospitales también se contempla la evaluación de sus servicios y el índice ocupacional, para reajustar los recursos materiales y humanos. En los casos donde no se justifique su permanencia, estos serán compactados o regionalizados. De hecho algunos resultados de la regionalización en provincias como Villa Clara, ya 
consideran la reducción de hospitales y su reconversión a otras funciones, como asilos de ancianos, o policlínicos con camas. ${ }^{13}$

Una importante actividad de reorganización es la referida al $24 \%$ de los municipios del país, donde existía una sola área de salud, o sea un solo policlínico, y también una Dirección de Salud Municipal, que ahora se funden, de forma que el policlínico asume la dirección y administración del sistema, eliminado la duplicación de funciones. El área de salud se define como el espacio territorial, con límites geográficos definidos, en el que reside una población que recibe atención integral de salud a través de un policlínico.

En el marco de las transformaciones, en los consultorios del médico y la enfermera de la familia, se redefine la población a atender, el número de los que brindarán servicios estables, y los recursos humanos necesarios en ellos, fijándose como máximo una población de hasta mil 500 habitantes en consultas y labores de terreno. Ello significa que del promedio de entre 120 a 160 familias que atender mantenido hasta el 2004, y el incremento producido a mediados de esta década, que llegó a ser de más de 600 familias, se producirá una reducción que en promedio representa la atención entre 300 a 400 familias por consultorio.

Llama la atención que la cifra de familias o individuos que pertenecen al área tributaria a un consultorio, o a un policlínico, aunque con certeza considerado, no tiene, que sepamos, definición de extensión, de distancia o de tiempo para acceder desde los asentamientos poblacionales a los servicios, componentes estos que se vuelven decisivos en algunos contextos, en particular en asentamientos rurales concentrados o dispersos, o de montaña, o en aquellos que han tenido impactos negativos en los procesos económicos más recientes, como el cierre de centrales azucareros y otros, donde los servicios de transporte están seriamente afectados.

Por otra parte, la revitalización de la medicina familiar con la reapertura de consultorios, ha sido divulgada por diferentes medios de prensa, retomándose distribuciones precedentes, con la consiguiente recuperación de algunas de las funciones que habían sido seriamente afectadas. Es esta sin dudas una importante vía para mejorar la disponibilidad de servicios de atención primaria y, potencialmente la satisfacción de la población con los servicios en la comunidad. ${ }^{14,15}$ En la Habana se conoce de un proceso similar, que en algunos casos toma como variante la división de la población a atender por el médico y la enfermera de la familia en dos consultorios funcionando dentro de una misma instalación.

Otra de las transformaciones se refiere al análisis de la racionalidad de los servicios de ambulancia, mediante cambios en la organización de los lugares donde estas radican (bases). Estas medidas que de forma inmediata reducen gastos e incrementan los controles, requieren de una cuidadosa vigilancia de su efectividad, dado que por lo general se trata de urgencias, y la demora en recibir el servicio es decisiva para la solución del problema de salud en cuestión.

La reorganización de los servicios de atención a las embarazadas en hogares maternos se basa en el índice de ocupación (gestantes por hogar materno), la distancia a policlínicos con camas y el acceso rápido al hospital ginecoobstétrico correspondiente, por lo que se prevé la reducción numérica de estos y con ello, sus estructuras de organización.

Algunos resultados de las transformaciones en marcha, muestran que en hogares maternos con capacidad solo para tres o cinco camas, existe en promedio 20 trabajadores y, algunos de estos hogares están cerca de unidades asistenciales que 
pueden garantizar la atención a las gestantes, ${ }^{16}$ la compactación de estos servicios ya ha provocado su reducción, como en el caso de la provincia de Villa Clara. ${ }^{13}$

Una observación de particular interés, es el reanálisis de las ofertas de estudio en algunas especialidades del sector, como la de tecnologías de la salud. Se reconoce que una de las causas del "excesivo número de trabajadores", se asocia a un incremento de la formación de recursos humanos en algunas especialidades, en especial de los formados en las carreras de tecnologías de la salud, lo cual es explicado por no haberse tenido en cuenta las necesidades de los territorios, para la oferta de matrículas en las instituciones correspondientes. Por otra parte, en algunos territorios, se constatan carencias de servicios de algunas especialidades, en lo fundamental médicas, que deben ser solucionadas.

Las transformaciones en marcha tienen una concepción integrada, y ello ha sido apuntado en el ámbito mundial como clave del éxito de las reformas en los sistemas de salud, dado que para ampliar la visión sobre estos sistemas, es necesario pensar, además de en sus componentes, tales como financiamiento, instituciones y tecnologías, pensar en sus interrelaciones. ${ }^{17}$

En las nuevas transformaciones de los servicios de salud en el país, la dimensión territorial tiene un especial significado y se asocia directamente a los múltiples factores objetivos y subjetivos, decisivos en la implementación y consolidación. Documentos y otras informaciones sobre el actual proceso de "Reorganización, concentración y regionalización de los servicios de salud" en el país, argumentan que las informaciones territoriales son tenidas en cuenta.

Este proceso íntimamente ligado al territorio, a la distribución de la población y las características de los asentamientos, debe haber definido además de una nueva cifra de población tributaria a las instituciones de nivel primario, las distancias a que quedarían estos servicios de cada asentamiento o barrio, y las condiciones de movilidad hacia los servicios relocalizados, o sea la accesibilidad real, con independencia de las distancias, factor que puede ser decisivo, dada las limitaciones del transporte público, más graves entre algunos asentamientos del país, y los precios del transporte privado que con seguridad no son accesibles a los ingresos de muchas familias.

En la teoría de la geografía de los servicios se considera que la distribución o redistribución de cualquiera de ellos, siempre deja en ventaja relativa a la población de un territorio, y en desventaja relativa a la de otro. Las distribuciones óptimas, en el caso de los servicios de salud, cuyo presupuesto es asumido por el Estado cubano de forma absoluta, están obligadas en este momento histórico a equilibrar la racionalidad económica, con una equidad, que es considerada sinónimo de igualdad estricto sensus por la población cubana.

En la revolución del sector de inicios de la década pasada, pueden haber sido decisivos criterios tales como las diferencias de contexto territorial poblacional, la localización en relación con otros servicios, la accesibilidad de la población a los servicios, la situación de salud, problemas y necesidades, entre otros, no obstante ellos fueron estructurados sin la presión de restricciones presupuestarias, o al menos sin la prioridad que la racionalidad económica impone en estos momentos.

A ello se integra, que a consecuencia de la invocación a una equidad desvirtuada, en el país se han implementado políticas, normas, o estrategias de acción en muchos sectores con una distribución territorial igualitaria. En el caso del sector de la salud, en la distribución de recursos y servicios, en especial los relacionados con tecnologías más modernas, es mucho más complicado definir parámetros de 
distribución equitativa, sin algunas renuncias, al fuerte componente humanitario y a la voluntad política, que sustentó las distribuciones anteriores. No se trata ahora de acercar la población a los servicios, sino de que la población se traslade a los servicios en los lugares donde les sea asignado.

La complejidad actual está atravesada no solo por la distribución geográfica de la población al interior de los municipios, como a los diferentes contextos económicos y sociales de estos. Aunque no se pretenda frustrar los derechos adquiridos, la "lejanía" relativa a la que quedarán algunos servicios, pudiera crear nuevas insatisfacciones.

Fomentar la conciencia de los riesgos del uso de equipos de alta tecnología, de la automedicación, o de los beneficios de la medicina natural y tradicional, aspectos considerados también en los lineamientos de la política del sector, no se consigue de forma rápida, se requieren acciones educativas y experiencias individuales o familiares favorables, vividas o creíbles en estos aspectos. Las menciones en diferentes medios, que con objetivos de corrección de un hábito adquirido, comunican estas informaciones a la población pueden tener efectos contrarios a los esperados. Es por ello, que ni siempre existe una relación directa entre incremento de los servicios y su calidad, o viceversa, o entre la calidad que se espera y la percibida por la población, máxime cuando está aún lastimado el elevado referencial de la calidad de la atención primaria en salud, construido durante más de dos décadas.

Deberán esperarse diferencias territoriales en los avances de las transformaciones, ni la calidad de la atención va a ser igual en todos los territorios, ni con los mismos servicios en dos territorios habrá igual satisfacción. Es evidente que en la capital del país, las expectativas, en especial de servicios de alta tecnología, son superiores, si comparadas con las de áreas montañosas del oriente cubano. Junto a la administración de salud, otras ciencias como la economía, la geografía, la sociología, la psicología, la antropología y la estadística, tendrán mucho que aportar en el abordaje complejo de los temas de distribución y equidad, tanto en el orden teórico cuanto práctico, no solo en el sector que nos ocupa, sino en el resto de los sectores que integran la salud pública.

\section{CONSI DERACI ONES FI NALES}

La propuesta de lineamientos de la política del sector de la salud, ${ }^{1}$ así como los procesos de reorganización, compactación y regionalización de los servicios de salud en marcha, indican que transitamos por nuevos cambios en el SNS cubano, que pretenden la reducción de los gastos en el sector a corto plazo, hecho ya constatado en los últimos años, con ahorros considerables en varias actividades.

La elevación de la calidad de los servicios, incluidos en los lineamientos de la política en salud, y de la satisfacción de la población, no tendrá resultados tan inmediatos, y de alguna forma estarán condicionados al mejoramiento de las condiciones de vida materiales y espirituales de los dos componentes humanos del sistema, que siempre participan en una doble condición: prestadores de serviciosciudadanos, y pacientes-ciudadanos.

Un arduo trabajo de atención a estos componentes, y una intensa articulación de ellos, con las complejas relaciones que se establecen entre financiamiento, organización y gestión, serán claves en los avances hacia la recuperación de los servicios, el perfeccionamiento de la atención y la permanencia de los logros 
asistenciales. La salud no es solo un sector específico de la administración pública, sino un objetivo social priorizado; en el caso de Cuba existen numerosas pruebas de los avances hacia este objetivo, que no pueden verse como cifras alcanzadas en indicadores relevantes a nivel mundial, sino como resultado de la aplicación de una estrategia y del despliegue de principios esenciales de salud pública. ${ }^{18}$

En el año 2006, Rojas Ochoa, uno de los más importantes sanitaristas del país, colocaba en entrevista publicada en la revista Temas, las grandes etapas de los modelos de atención a la salud en Cuba:

...una inicial, todavía no muy caracterizada del policlínico integral; la del policlínico comunitario; y la medicina familiar que parece estar llegando a lo que le ha pasado a los anteriores, a un momento que no satisface todas las demandas. Se está agotando el modelo, y ahora no sabemos cómo emergerá el nuevo. ${ }^{19}$

A la solicitud del periodista, adelanta algunas hipótesis sobre lo que podría venir:

Yo aspiraría, - no sé si es lo que va a venir o no- a algo ligado estrechamente a la formación del médico que atienda más a la dinámica social de la salud y de la enfermedad. Más promoción de salud...una mayor atención a los problemas sociales que tienen que ver con la salud del hombre... es preciso que el nuevo modelo no le rinda tanto culto a la alta tecnología, que nos asentemos un poco en los viejos procedimientos, tan eficaces, como la clínica. ${ }^{19}$

Más recientemente, en una conducta totalmente proactiva, Rojas Ochoa, escribe diez "rectificaciones necesarias", ${ }^{20}$ coincidentes casi totalmente con las medidas de transformación del SNS que meses después propone el Ministerio de Salud Pública.

Las "rectificaciones necesarias" de Rojas Ochoa, incluidas en el documento que bajo el titulo "Transformaciones necesarias del Sistema de Salud Pública"12 hemos comentado, diferenciada en lo fundamental, por la importancia que este salubrista le otorga a la necesidad de la estimulación a los que desempeñan sus tareas cotidianas de forma heroica, tanto en misiones fuera del país como en Cuba y marcada prioridad a la formación y perfeccionamiento de los recursos humanos en salud.

Se destaca en esta propuesta, la prioridad a los problemas sociales de la población y a la estimulación de los trabajadores del sector, entre otros aspectos.

El fortalecimiento de la promoción de salud, también incluido en los lineamientos políticos del sector, y el conjunto de acciones a ella asociada, exige tal vez más que en otros sectores, la estrecha y armónica relación entre -prestadores y beneficiarios-. Es bien conocido que las políticas del sector no son suficientes para proteger, mejorar, ni mantener la salud de la población, y necesitan de la integración de numerosas políticas sectoriales, y de la bien conocida y aún por perfeccionar intersectorialidad, decisiva para mejorar la salud social.

La esencia humanista del sistema social cubano no variará, como no lo ha hecho en otras circunstancias difíciles, y junto a otros tantos objetivos en especial de actualización del modelo económico, con certeza se procurará preservar y mejorar la salud de la población. Los avances en la nutrición, la vivienda e higiene adecuada, la disminución del estrés, la satisfacción con la ocupación, y tantos otros componentes que condicionan la construcción social de la salud, se conseguirán en 
la medida en que se avance en la instrumentación de muchos de los lineamientos políticos y económicos del país, y no solo en los referidos al sector de la salud.

Mantenida la gratuidad y la cobertura universal, el SNS se enfrenta en las actuales circunstancias a la conciliación entre racionalidad económica y calidad, pero en paralelo necesita reelaborar la concepción de la equidad distributiva de recursos y servicios, y favorecer que la población reconstruya sus percepciones y referenciales sobre la atención a la salud.

\section{REFERENCI AS BI BLI OGRÁFICAS}

1. Partido Comunista de Cuba. Lineamientos de la política económica y social del Partido y la Revolución. 18 de abril del 2011:24.

2. Dirección Nacional de Estadísticas. Sistema de Salud [Internet]. La Habana: Ministerio de Salud Pública; Dic 1998 [citado 25 Dic 2010]. Disponible en: http://www.sld.cu/sistema_de_salud/estrategias.html

3. Castro R. Discurso pronunciado en la clausura de la Asamblea Nacional del Poder Popular, en el Palacio de Convenciones, el 20 de diciembre del 2009. Periódico Granma [Internet]. 21 Dic de 2009 [citado 25 Dic 2010]. Disponible en: http://www.cuba.cu/gobierno/rauldiscursos/2009/esp/r201209e.html

4. Chang M, Alemán ML, Cañizares MP, I barra AM. Satisfacción de los pacientes con la atención médica. Rev Cubana Med Gen Integr [Internet]. 1999 [citado 28 Nov 2011]; 15(5):541-7 Disponible http://scielo.sld.cu/scielo.php?script=sci_arttext\&pid=S0864

en: $\underline{21251999000500009 \& \operatorname{lng}=\mathrm{es} \& \mathrm{nrm}=\mathrm{iso \& t} \operatorname{lng}=\mathrm{es}}$

5. Suárez JJ. El sistema de salud en Cuba. Desafíos hacia el año 2000. Rev Cubana Salud Pública [Internet]. 1997 [citado 25 Dic 2010];23(1-2):5-16. Disponible en: http://scielo.sld.cu/scielo.php?script=sci arttext\&pid=S0864-

$\underline{34661997000100001 \& \operatorname{lng}=e s \& n r m=i s o \& t \operatorname{lng}=e s}$

6. Luna EM, Sierra DP, Pérez Gandul SL. La transformación del policlínico en Cuba de cara al siglo Xxı. Rev Cubana Med Gen Integr [Internet]. 2009 [citado 25 Dic 2010];25(2). Disponible en: http://scielo.sld.cu/scielo.php?script=sci arttext\&pid=S0864$\underline{21252009000200016 \& \operatorname{lng}=e s \& n r m=i s o \& t \operatorname{lng}=e s}$

7. García J. Sobrevivirán los niveles de atención a la revolución de la salud pública cubana. Rev Cubana Salud Pública [Internet]. 2006 [citado 25 Dic 2010];32(1). Disponible en: $\quad$ http://scielo.sld.cu/scielo.php?script=sci_arttext\&pid=S086434662006000100014\&lng=es\&nrm=iso\&tlng=es

8. Terrero A. Otra vuelta de rosca. Revista Bohemia, 24 marzo 2008.

9. Edith D, Terrero A. Salud. ¿Dónde están los médicos? Revista Bohemia Edición No. 6, del 16 de marzo de 2007.

10. Ministerio de Salud Pública. Anuario Estadístico de Salud 2009. La Habana: MI NSAP; 2010. 
11. Ministerio de Salud Pública. Anuario Estadístico de Salud 2010. La Habana: MI NSAP; 2011.

12. Ministerio de Salud Pública. Transformaciones necesarias en el Sistema de Salud Pública. La Habana: MINSAP; 2010.

13.González R. Emprenden reordenamiento del sistema de Salud en Villa Clara. Periódico Vanguardia, 9 noviembre de 2010.

14. Leyva DF. Revitalizada la medicina familiar en Cienfuegos con la reapertura de nuevos consultorios. Perlavisión, 6 diciembre de 2010.

15. González R. Declarados listos los consultorios del médico y la enfermera de la familia en Villa Clara. Periódico Vanguardia, 3 diciembre de 2010.

16. De la Osa. Hacia la racionalidad en la provincia de La Habana. Periódico Granma [Internet]. 5 Octubre de 2010 [citado 28 Nov 2011]. Disponible en: http://www.granma.cubaweb.cu/2010/10/05/nacional/artic01.html

17. Frenk J. The Global Health System: Strengthening National Health Systems as the Next Step for Global Progress. PLoS Med [Internet]. Jan 2010 [citado 28 Nov 2011];7(1). e1000089. doi:10.1371/journal.pmed.1000089. Disponible en: http://www. plosmedicine.org/article/info\% 3Adoi\% 2F10.1371\%2Fjournal.pmed. 100 $\underline{0089 ; i}$

18. Cooper RC, Kenelly JF, Orduñez GP. Health in Cuba. Internat J Epidemiol. 2006; 35: 817-24.

19. Rodríguez Oliva LI. Modelos de salud en Cuba. Habla un protagonista. Entrevista a Francisco Rojas Ochoa. Revista Temas. 2006; (47):35-43.

20. Rojas Ochoa F. Rectificaciones necesarias [Internet]. 2010 [citado 6 Sept 2010]. Disponible en: http://articulos.sld.cu/revsalud/category/primero_en_la_web/

Recibido: 16 de septiembre de 2011.

Aprobado: 29 de septiembre de 2011.

Luisa Iñiguez Rojas. Centro de Estudios de Salud y Bienestar Humano. Universidad de La Habana. La Habana, Cuba.

Correo electrónico: cesbh@matcom.uh.cu, luisab@lab.matcom.uh.cu,

iniguezrojas@yahoo.com 